\title{
MÉTODOS DE AMOSTRAGEM DE ÁREA VARIÁVEL PARA A REGENERAÇÃO NATURAL DE Eremanthus erythropappus (DC.) MacLeish
}

\author{
Emanuel José Gomes de Araújo ${ }^{1}$, Sylvio Péllico Netto², Vinícius Augusto Morais ${ }^{3}$, Hassan Camil David ${ }^{4}$, \\ Rafaella De Angeli Curto ${ }^{5}$, José Roberto Soares Scolforo ${ }^{6}$

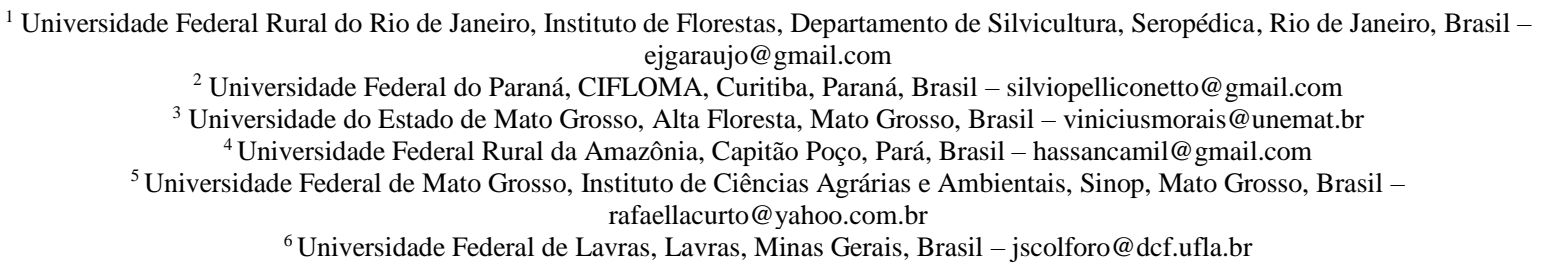

Recebido para publicação: 09/10/2017 - Aceito para publicação: 19/12/2017

\begin{abstract}
Resumo
O objetivo deste trabalho foi aplicar e avaliar o desempenho de métodos de amostragem de área variável em regeneração natural da espécie candeia em áreas submetidas ao sistema de manejo com porta sementes. Os dados foram coletados em 46 Unidades de Estudos com regeneração natural da espécie. Foram simuladas variações estruturais dos métodos de amostragem de Strand e Prodan para estimar o número de plantas por hectare e a altura média. Os valores estimados foram comparados com o valor de referência por meio de regressão, do coeficiente de correlação Pearson e do índice de concordância de Willmott. O desempenho foi avaliado pela associação da correlação de Pearson com o índice de Willmott. O teste não paramétrico de Kruskal-Wallis foi aplicado para verificar diferenças estatísticas entre as médias estimadas e a referencial. O método de Strand com linhas de 4 e $5 \mathrm{~m}$ foi preciso e exato na estimativa da densidade e da altura média. O método de Prodan superestimou a densidade, mas foi preciso para estimar a altura média. Conclui-se que o método de amostragem de Strand, utilizando linha de $4 \mathrm{~m}$ de comprimento, pode ser utilizado para amostrar a regeneração natural da espécie candeia, que apresenta padrão espacial agregado.
\end{abstract}

Palavras-chave: Método de Strand, método de Prodan, agregação.

\begin{abstract}
Sampling methods of variable area applied to natural regeneration of Eremanthus erythropappus (DC.) MacLeish. The objective of this study was to apply and assess the performance of sampling methods of variable area in natural regeneration of the species Eremanthus erythropappus in areas subject to management system with seed-trees. Data were collected in 46 Study Units with natural regeneration of the species. Structural variations of Strand's and Prodan's sampling methods were simulated to estimate the number of plants per hectare and average height. The estimated values for each case were compared to a given reference value by means of regression, Pearson's coefficient of correlation, and Willmott's concordance index. The performance was assessed by the association of Pearson's correlation with Willmott's index. The nonparametric Kruskal-Wallis' test was applied to verify significant differences between the estimated and referential means. Strand's sampling method with lines of 4 and $5 \mathrm{~m}$ was precise and accurate for estimating density and average height. Prodan's sampling method overestimated density, but it was accurate to estimate height. We concluded that Strand's sampling method, using a line of $4 \mathrm{~m}$ in length, can be used for sampling natural regeneration of E. erythropappus, which presents aggregated spatial pattern.

Keywords: Strand's sampling method, Prodan's sampling method, aggregation.
\end{abstract}

\section{INTRODUÇÃO}

A candeia, Eremanthus erythropappus (DC.) MacLeish, é uma espécie florestal de múltiplos usos. Sua madeira é muito utilizada como moirão de cerca, em decorrência da alta durabilidade, e para a extração de óleo essencial, cujo princípio ativo é o $\alpha$-bisabolol, empregado na fabricação de medicamentos e cosméticos. Estes possuem alta demanda no mercado e são responsáveis por valorizar o óleo essencial produzido pela candeia (SCOLFORO et al., 2012; SANTOS et al., 2008), destacando-se o potencial econômico da espécie

FLORESTA, Curitiba, PR, v. 48, n. 2, p. 265-276, abr/jun. 2018.

Araujo. E. J. G et.al.

ISSN eletrônico 1982-4688

DOI: $10.5380 /$ rf.v48 i 2.55726 
(MORI et al., 2010; OLIVEIRA et al., 2011; MELO et al., 2012). Diante disso, a candeia foi explorada de forma desordenada por muito tempo. Na última década, surgiram os primeiros estudos com o manejo da candeia, em especial aqueles voltados para a regeneração natural em áreas submetidas ao manejo sustentável (SCOLFORO et al., 2012; OLIVEIRA et al., 2011; PAES et al., 2010; PÉREZ et al., 2004; ARAÚJO et al., 2012; SILVA et al., 2008; SCOLFORO et al., 2008). Porém, existe uma lacuna quanto à aplicação de métodos de amostragem para a espécie, principalmente para a regeneração natural, devido a sua alta variabilidade, o que consiste em uma das dificuldades nos inventários florestais.

O maior objetivo da teoria de amostragem é alicerçar as metodologias que propiciam eficiência, com alta precisão e exatidão, gerando baixo custo (KERSHAW JR. et al., 2017; SANQUETTA et al., 2014). Vários métodos de amostragem podem ser utilizados em inventários florestais, sendo eles de área fixa e de áreas variáveis. Dentre os métodos de áreas variáveis podem ser citados os propostos por Strand (1958) e Prodan (1968). O método da linha, ou método de Strand, é uma aplicação da técnica de contagem angular (LOETSCH et al., 1973). Prodan (1968) desenvolveu uma metodologia que considera a distância da sexta planta mais próxima do ponto amostral. Ambos foram descritos por Péllico Netto e Brena (1997).

A escolha do método de amostragem depende da finalidade do inventário, da espécie, das condições locais e das características da população. Além disso, deve-se considerar o padrão de distribuição espacial das plantas. Kleinn e Vilcko (2006) destacam que essa característica influencia na amostragem, principalmente para o método da k-árvore, quando o padrão espacial das árvores na floresta é agregado. Diante do exposto, o objetivo deste trabalho foi aplicar e avaliar o desempenho de métodos de amostragem de área variável para regeneração natural da espécie Eremanthus erythropappus (DC.) MacLeish.

\section{MATERIAL E MÉTODOS}

\section{Área de estudo e banco de dados}

Os dados foram coletados em 46 áreas, aqui denominadas de Unidades de Estudo (UEs), com regeneração natural de candeia (Eremanthus erythropappus). As UEs estão distribuídas na região sul do estado de Minas Gerais, entre as coordenadas $22^{\circ} 16^{\prime} 48^{\prime \prime} \mathrm{S}, 45^{\circ} 24^{\prime} 20^{\prime \prime}$ W e $21^{\circ} 59^{\prime} 04^{\prime}$ ' S, 4445'16' W (ARAÚJO et al., 2012). As áreas de ocorrência natural da candeia são de encraves, entre matas e campos abertos, caracterizadas por apresentar clima, segundo a classificação de Köppen, mesotérmico úmido do tipo Cwb, tropical de altitude e com verões suaves. A altitude média varia de $950 \mathrm{~m}$ a $1.750 \mathrm{~m}$, com precipitação média anual entre $1.600 \mathrm{~mm}$ e $1.700 \mathrm{~mm}$ e temperatura média anual variando de $16^{\circ} \mathrm{C}$ a $17^{\circ} \mathrm{C}$. Os solos predominantes são Cambissolo Húmico, Neossolo Litólico e Argissolo Vermelho (CARVALHO et al., 2008).

Cada UE possui área de $300 \mathrm{~m}^{2}$ e formato de um polígono regular de 12 lados (ARAÚJO et al., 2012). Para obter a média referencial, que foi considerada como estimador do parâmetro populacional, em cada UE, foi efetuado o censo das plantas regenerantes, no qual foi mensurada a variável altura total $(h)$ de cada uma. Considerou-se como plantas regenerantes todas aquelas com altura $\geq 5 \mathrm{~cm}$ e diâmetro a $1,30 \mathrm{~m}$ do solo $\leq 5 \mathrm{~cm}$. Além disso, foram obtidas as coordenadas cartesianas de cada planta mensurada por meio de croqui milimetrado. A coordenada geográfica no centro de cada polígono, obtida por meio de GPS Garmin, foi considerada como origem do plano cartesiano, permitindo o georreferenciamento de todas as plantas em todos os polígonos. Isso foi feito com a finalidade de simular os métodos de amostragem de Strand e Prodan.

\section{Método de Amostragem de Strand}

O primeiro método aplicado nas 46 UE foi o desenvolvido por Strand (1958), que considera a seleção das plantas na unidade amostral com proporcionalidade à altura para obtenção da estimativa de densidade. Sua abordagem é feita com base em uma unidade amostral constituída de uma linha de comprimento L tomada dentro da população, ao longo da qual se enumera todas as plantas que atendem ao critério de inclusão. Para esse estudo, o fator de proporcionalidade $\left(\mathrm{k}_{2}\right)$ foi igual a um, ou seja, incluiu-se na amostragem todas as plantas regenerantes com altura total $(\mathrm{h})$ igual ou superior à sua distância $(\mathrm{d})$ até a linha $\mathrm{L}$.

Em cada UE, sistematizou-se 4 linhas de comprimento (L), variando de 1 a $5 \mathrm{~m}$. Cada comprimento de linha caracterizou uma variação estrutural do método original (STRAND, 1958). Assim, foram aplicadas cinco variações denominadas de $\mathrm{L}=1 \mathrm{~m}, \mathrm{~L}=2 \mathrm{~m}, \mathrm{~L}=3 \mathrm{~m}, \mathrm{~L}=4 \mathrm{~m}$ e L=5 m, com amostragem das plantas realizada somente do lado esquerdo da linha. Por fím, estimou-se a densidade $\left(\mathrm{N} \cdot \mathrm{ha}^{-1}\right)$ e a altura média $(\overline{\mathrm{H}})$ das 46 UEs para comparação com as médias referenciais de 3.167 plantas.ha ${ }^{-1}$ e $0,79 \mathrm{~m}$, respectivamente. A estimativa do número de plantas por hectare de cada UE (1) foi feita com base nos fundamentos teóricos descritos por Péllico Netto e Brena (1997). 


$$
\text { N.ha }{ }^{-1}=\frac{10.000}{\mathrm{~L}} \sum_{\mathrm{i}=1}^{\mathrm{m}}\left(\frac{1}{\mathrm{~h}_{\mathrm{i}}}\right)
$$

Em que: $\mathrm{N} \cdot \mathrm{ha}^{-1}=$ número de plantas por hectare; $\mathrm{L}=$ comprimento da linha de amostragem; $\mathrm{m}=$ número de plantas amostradas; $h_{i}=$ altura da i-ésima planta amostrada.

Para estimar a altura média, ainda não há uma formulação teórica específica para o método de amostragem de Strand, sendo necessário o seu desenvolvimento. Para isso, seguiu-se a concepção de Hirata (1955), em que a seleção de plantas é feita com proporcionalidade a altura e as unidades amostrais são compostas por pontos de Bitterlich. A descrição da altura média de Hirata está apresentada em Péllico Netto e Brena (1997). Com base nisso, demonstramos, a seguir, o desenvolvimento para o cálculo da altura média estimada pelo método de Strand.

Considerando a amostragem de plantas, com probabilidade proporcional à altura ao longo de uma linha de comprimento L, que compreende uma unidade amostral, tem-se:

$$
\operatorname{tg} \beta=\frac{h_{i}}{R_{i}}=k_{2}
$$

Considera-se $\mathrm{k}_{2}=1$, ou seja, o critério de inclusão é $\mathrm{h}_{\mathrm{i}} \geq \mathrm{R}_{\mathrm{i}}$, e $\mathrm{R}_{\mathrm{i}}$ é igual à distância $\mathrm{d}_{\mathrm{i}}$ da i-ésima planta até a linha de amostragem (L). Isolando-se $\mathrm{R}_{\mathrm{i}}$, tem-se:

$$
\mathrm{R}_{\mathrm{i}}=\frac{\mathrm{h}_{\mathrm{i}}}{\operatorname{tg} \beta}
$$

Para cada planta amostrada na linha de comprimento $L$, sua área $\left(A_{i}\right)$ de ocupação será $A_{i}=R_{i} L$ e a probabilidade $\left(\mathrm{p}_{\mathrm{i}}\right)$ de sua ocorrência na área amostrada será:

$$
\mathrm{p}_{\mathrm{i}}=\frac{1}{\mathrm{R}_{\mathrm{i}} \mathrm{L}}
$$

Portanto, se em cada unidade amostral (linha de amostragem) forem amostrados m plantas (árvores ou regenerações), então $m$ plantas estarão contidas na área $\mathrm{Q}$, que é representada pelo somatório das áreas $\mathrm{A}_{\mathrm{i}}$ de cada planta e, matematicamente, pode ser descrito da seguinte maneira:

$$
\mathrm{Q}=\sum_{\mathrm{i}=1}^{\mathrm{m}} \mathrm{A}_{\mathrm{i}}=\sum_{\mathrm{i}=1}^{\mathrm{m}} \mathrm{R}_{\mathrm{i}} \mathrm{L}=\mathrm{L} \sum_{\mathrm{i}=1}^{\mathrm{m}} \mathrm{R}_{\mathrm{i}} \quad \begin{aligned}
& \text { Para estimar o número de } \\
& \text { plantas por hectare (n), tem-se: }
\end{aligned}
$$

Substituindo $\mathrm{R}_{\mathrm{i}}$ por $\frac{\mathrm{h}_{\mathrm{i}}}{\operatorname{tg} \beta}$, tem-se:

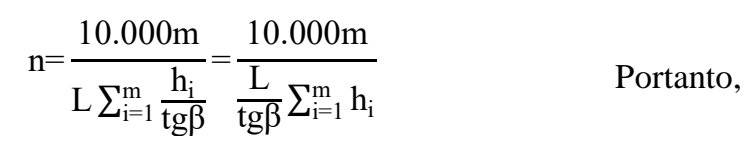

$$
\mathrm{n}=\frac{10.000 \mathrm{~m}}{\mathrm{Q}}=\frac{10.000 \mathrm{~m}}{\mathrm{~L} \sum_{\mathrm{i}=1}^{\mathrm{m}} \mathrm{R}_{\mathrm{i}}}
$$

Para $\mathrm{h}_{\mathrm{i}}=\mathrm{R}_{\mathrm{i}}$, temos $\operatorname{tg} \beta=\mathrm{k}_{2}=1 ; \log \mathrm{O}$ :

$$
\mathrm{n}=\frac{10.000 \mathrm{~m}}{\mathrm{~L} \sum_{\mathrm{i}=1}^{\mathrm{m}} \mathrm{h}_{\mathrm{i}}}=\frac{10.000}{\mathrm{~L} \frac{\sum_{\mathrm{i}=1}^{\mathrm{m}} \mathrm{h}_{\mathrm{i}}}{\mathrm{m}}}=\frac{10.000}{\mathrm{~L} \overline{\mathrm{h}}} \quad \text { Finalmente, (10) } \quad \overline{\mathrm{h}}=\frac{10.000}{\mathrm{Ln}} \quad \text { Com n=N.ha }{ }^{-1}
$$

Se a amostragem for efetuada em u unidades amostrais (ua) que representam u linhas de comprimento L, entã,o em cada ua, tem-se um valor de $\overline{\mathrm{h}}_{\mathrm{j}}$ e $\mathrm{n}_{\mathrm{j}}$, tal que $\mathrm{j}=1,2,3, \ldots$, u e a altura média das ua será obtida por:

$$
\begin{array}{r}
\overline{\mathrm{h}}_{\mathrm{u}}=\frac{\sum_{\mathrm{i}=1}^{\mathrm{u}} \overline{\mathrm{h}}_{\mathrm{j}}}{\mathrm{u}}=\frac{1}{\mathrm{u}} \sum_{\mathrm{i}=1}^{\mathrm{u}} \overline{\mathrm{h}}_{\mathrm{j}}=\frac{1}{\mathrm{u}} \sum_{\mathrm{i}=1}^{\mathrm{u}} \frac{10.000}{\mathrm{Ln}_{\mathrm{j}}} \quad \text { Finalmente, } \\
\overline{\mathrm{h}}_{\mathrm{u}}=\frac{10.000}{\mathrm{uL}}\left(\frac{1}{\mathrm{n}_{1}}+\ldots+\frac{1}{\mathrm{n}_{\mathrm{u}}}\right)=\overline{\mathrm{H}}
\end{array}
$$$$
\overline{\mathrm{h}}_{\mathrm{u}}=\frac{1}{\mathrm{u}}\left(\frac{10.000}{\mathrm{Ln}_{1}}+\ldots+\frac{10.000}{\operatorname{Ln}_{\mathrm{u}}}\right)
$$ 
Os valores de $n_{j}(14)$ são obtidos pela amostragem proporcional à altura, descrita em Péllico Netto e Brena (1997) e apresentada a seguir:

$$
\mathrm{n}_{\mathrm{j}}=\mathrm{N} \cdot \mathrm{ha}^{-1}=\frac{10.000}{\mathrm{~L}} \sum_{\mathrm{i}=1}^{\mathrm{m}}\left(\frac{1}{\mathrm{~h}_{\mathrm{i}}}\right) \quad \text { Com } \mathrm{i}=1,2,3, \ldots, \mathrm{m}
$$

\section{Método de Amostragem de Prodan}

Também, aplicou-se o método proposto por Prodan (1968), que considera como critério de inclusão de uma planta na amostragem a sua distância ao ponto amostral e, portanto, se faz com proporcionalidade à distância. O método original é denominado como das seis árvores, pois considera a distância da sexta árvore até o ponto amostral de referência (PÉLLICO NETTO; BRENA, 1997). Este trabalho considerou a distância até a trigésima planta mais próxima do ponto amostral, configurando o método original mais 29 variações.

Para as simulações das variações estruturais do método de Prodan, foram consideradas duas premissas: a distância da $30^{\mathrm{a}}$ planta até o ponto amostral não ser superior a distância do ponto amostral até os limites da UE para não caracterizar estimativa fora dos limites da UE; e dois pontos amostrais dentro da UE não deverão se sobrepor quanto a sua área de abrangência para assegurar independência das UEs. Sendo assim, das 46 UEs iniciais, apenas 21 atenderam aos dois critérios. Em cada UE selecionada, um ponto amostral foi escolhido aleatoriamente e, a partir daí, foram determinados os raios $\left(\mathrm{R}_{\mathrm{i}}\right)$, que indicam a distância da i-ésima planta mais próxima desses pontos, com i variando de 1 a 30. Finalmente, estimou-se a densidade (N.ha ${ }^{-1}$ ) e a altura média $(\overline{\mathrm{H}})$ das 21 UE para comparar com as médias referenciais de 5.803 plantas.ha $^{-1}$ e 0,82 m, respectivamente. Esse procedimento foi simulado 10.000 vezes para obtenção de estimativas confiáveis. A formulação matemática para estimar o número de regenerações por hectare (15), em cada UE, para as variações do método é apresentada a seguir:

$$
\mathrm{N} \cdot \mathrm{ha}^{-1}=\frac{\mathrm{f}(10.000)}{\pi \cdot \mathrm{R}_{\mathrm{i}}^{2}}
$$

Em que: $\mathrm{N} \cdot \mathrm{ha}^{-1}=$ número de plantas por hectare; $\pi=$ constante pi; $\mathrm{R}_{\mathrm{i}}=$ raio (distância) da i-ésima planta até o ponto de amostragem $(\mathrm{i}=1,2,3, \ldots, 30) ; \mathrm{f}=$ fator aplicado para a i-ésima planta distanciada do ponto amostral, contabilizando esta como sendo meia planta $\left(i_{1}=0,5 ; i_{2}=1,5 ; i_{3}=2,5 ; \ldots ; i_{30}=29,5\right)$, visto que o $R_{i}$, teoricamente, é a distância do ponto central da amostragem até o meio do caule da i-ésima planta.

A altura média em cada UE (16), em cada variação estrutural do método de amostragem, foi estimada por meio da média aritmética da altura observada da i-ésima planta amostrada e limítrofe da unidade amostral.

$$
\overline{\mathrm{H}}=\frac{\sum_{\mathrm{i}=1}^{\mathrm{m}}\left(\mathrm{h}_{\mathrm{i}}\right)}{\mathrm{m}}
$$

Em que: $\overline{\mathrm{H}}=$ altura média estimada da UE; $\mathrm{m}=$ número de pontos de amostragem na $\mathrm{UE}(\mathrm{m}=1,2, \ldots, 30) ; \mathrm{h}_{\mathrm{i}}=$ altura da i-ésima planta amostrada e limítrofe da unidade amostral $(\mathrm{i}=1,2, \ldots, 30)$.

\section{Estatística}

Em cada método, buscou-se avaliar a precisão e exatidão na estimativa das variáveis: número de plantas por hectare (densidade em N.ha ${ }^{-1}$ ) e altura total média $(\overline{\mathrm{H}})$. Todas as análises e simulações foram realizadas com auxílio dos softwares Matlab Starter Application e R (R DEVELOPMENT CORE TEAM, 2011).

O método de Strand foi composto por 5 tratamentos (método $S$ ), com 46 repetições cada, e o método de Prodan por 30 tratamentos, com 21 repetições cada. Aplicou-se o teste de Bartlett, a 95\% de probabilidade, para verificar a homogeneidade da variância da amostra obtida em cada tratamento. Rejeitada a hipótese de homogeneidade da variância, aplicou-se o teste não paramétrico de Kruskal-Wallis (PIMENTEL-GOMES, 2009), a 95\% de probabilidade, para avaliar diferença significativa entre as médias estimadas em cada tratamento e a média referencial, estimador mais exato do valor paramétrico, a partir de comparações múltiplas.

\section{Precisão, Exatidão e Desempenho}

Para cada variação dos métodos de Strand e Prodan, foi calculado o erro médio absoluto (17), conforme apresentado a seguir: 


$$
\mathrm{EMA}=\frac{\sum_{\mathrm{i}=1}^{\mathrm{n}}\left|\hat{\mathrm{y}}_{\mathrm{i}}-\mathrm{y}_{\mathrm{i}}\right|}{\mathrm{n}}
$$

Em que: EMA = erro médio absoluto; $\mathrm{y}_{\mathrm{i}}=\mathrm{i}$-ésimo valor observado; $\hat{\mathrm{y}}_{\mathrm{i}}=\mathrm{i}$-ésimo valor estimado; $\mathrm{n}=$ número de observações.

Além disso, fez-se o ajuste de uma regressão linear simples $\left(y=\beta_{0}+\beta_{1} x+e_{i}\right)$, com os valores estimados em cada UE em função dos seus respectivos valores referenciais. Avaliou-se a significância dos coeficientes da regressão ajustada para retratar a tendência no gráfico. As hipóteses $\mathrm{H}_{0}: \beta_{0}=0$ e $\mathrm{H}_{0}: \beta_{1}=1$ foram testadas com o teste $t$, a $95 \%$ de probabilidade. Quando tais hipóteses de nulidade são aceitas, verifica-se que os valores estimados são próximos dos referenciais, retratando uma linha de regressão com inclinação de $45^{\circ}\left(\beta_{1}=1\right)$ e passando pela origem $\left(\beta_{0}=0\right)$, o que caracteriza exatidão. Ainda, observaram-se os valores de coeficiente de determinação $\left(\mathrm{R}^{2}\right)$ e de correlação de Pearson (R) da regressão para retratar a precisão.

Aplicou-se o índice de exatidão de Willmott (1981) (18), que mede o grau de concordância entre os valores estimados e os paramétricos, variando de 0 a 1 . Serão tão melhores quanto mais próximos estiverem de 1.

$$
\mathrm{W}=1-\frac{\sum_{\mathrm{i}=1}^{\mathrm{n}}\left(\hat{\mathrm{y}}_{\mathrm{i}}-\mathrm{y}_{\mathrm{i}}\right)^{2}}{\sum_{\mathrm{i}=1}^{\mathrm{n}}\left(\left|\hat{\mathrm{y}}_{\mathrm{i}}-\overline{\mathrm{y}}\right|+\left|\mathrm{y}_{\mathrm{i}}-\overline{\mathrm{y}}\right|\right)^{2}}
$$

Em que: $\mathrm{W}=$ índice de Willmott; $\mathrm{y}_{\mathrm{i}}=\mathrm{i}$-ésimo valor observado; $\hat{\mathrm{y}}_{\mathrm{i}}=\mathrm{i}$-ésimo valor estimado; $\overline{\mathrm{y}}=$ média dos valores observados; $\mathrm{n}=$ número de observações.

Associando os valores de correlação da regressão (precisão) com os de concordância de Willmott (exatidão), tem-se o índice de desempenho (c), por meio da seguinte relação: $\mathrm{c}=\mathrm{RW}$. O critério de interpretação do desempenho foi adaptado para este trabalho e caracterizou-se como péssimo $(0 \leq \mathrm{c} \leq 0,20)$, ruim $(0,20<\mathrm{c} \leq 0,40)$, regular $(0,40<\mathrm{c} \leq 0,60)$, bom $(0,60<\mathrm{c} \leq 0,80)$ e ótimo $(0,80<\mathrm{c} \leq 1,0)$.

\section{RESULTADOS}

O teste de Bartlett foi significativo (p-valor $<0,05$ ), sendo necessária a aplicação do teste de KruskalWallis, a 95\% de probabilidade, para comparar as médias estimadas em cada método com a média referencial nos dois métodos de amostragem. O teste de Kruskal-Wallis foi não significativo (p-valor > 0,05), indicando que as médias estimadas de densidade e altura total para cada tamanho de linha foram iguais à média referencial (Tabela 1). A média referencial se encontra entre os limites do intervalo de confiança, a 95\% de probabilidade, paras os comprimentos de linha 2, 3, 4 e $5 \mathrm{~m}$, demonstrando que as variações do método de Strand apresentaram estimativas confiáveis para as variáveis densidade (plantas.ha ${ }^{-1}$ ) e altura média. A linha com $1 \mathrm{~m}$ de comprimento não foi exata para estimar tais variáveis. Na Figura 1, estão apresentados os valores estimados em função dos referenciais, em cada UE, para a variável densidade (número de plantas.ha ${ }^{-1}$ ) e altura média. A Tabela 2 apresenta o desempenho das variações do método de Strand para as variáveis densidade e altura média.

Tabela 1. Estatísticas da amostragem das variações do método de Strand para as variáveis densidade e altura média.

\begin{tabular}{|c|c|c|c|c|c|c|}
\hline \multirow{2}{*}{ Método Strand } & Média & Mínimo & Máximo & Desvio & Intervalo & Referencial \\
\hline & \multicolumn{6}{|c|}{ Densidade (Número de Plantas Por Hectare) } \\
\hline $\mathrm{L}=1 \mathrm{~m}$ & 4.638 & 0 & 43.879 & 1.204 & $3.434 \leq \overline{\mathrm{R}} \leq 5.841$ & \\
\hline $\mathrm{L}=2 \mathrm{~m}$ & 3.822 & 0 & 27.739 & 863 & $2.959 \leq \overline{\mathrm{R}} \leq 4.685$ & \\
\hline $\mathrm{L}=3 \mathrm{~m}$ & 3.513 & 0 & 23.894 & 755 & $2.757 \leq \overline{\mathrm{R}} \leq 4.268$ & 3.167 \\
\hline $\mathrm{L}=4 \mathrm{~m}$ & 3.268 & 0 & 21.090 & 706 & $2.561 \leq \overline{\mathrm{R}} \leq 3.974$ & \\
\hline \multirow[t]{2}{*}{$\mathrm{L}=5 \mathrm{~m}$} & 3.262 & 0 & 20.389 & 698 & $2.563 \leq \overline{\mathrm{R}} \leq 3.960$ & \\
\hline & \multicolumn{6}{|c|}{ Altura (m) } \\
\hline $\mathrm{L}=1 \mathrm{~m}$ & 1,1017 & 0,1854 & 3,5000 & 0,2042 & $0,89 \leq \overline{\mathrm{R}} \leq 1,30$ & \\
\hline $\mathrm{L}=2 \mathrm{~m}$ & 0,9127 & 0,0945 & 2,7750 & 0,1294 & $0,78 \leq \overline{\mathrm{R}} \leq 1,04$ & 0,79 \\
\hline $\mathrm{L}=3 \mathrm{~m}$ & 0,8870 & 0,1051 & 2,7750 & 0,1162 & $0,77 \leq \overline{\mathrm{R}} \leq 1,00$ & \\
\hline
\end{tabular}
Table 1. Descriptive statistics for the variations of Strand sampling method to estimate tree density and mean height.

FLORESTA, Curitiba, PR, v. 48, n. 2, p. 265-276, abr/jun. 2018. 


$\begin{array}{llllll}\mathrm{L}=4 \mathrm{~m} & 0,8338 & 0,1592 & 2,7750 & 0,1078 & 0,72 \leq \overline{\mathrm{R}} \leq 0,94 \\ \mathrm{~L}=5 \mathrm{~m} & 0,7156 & 0,1179 & 2,7750 & 0,1067 & 0,60 \leq \overline{\mathrm{R}} \leq 0,82\end{array}$
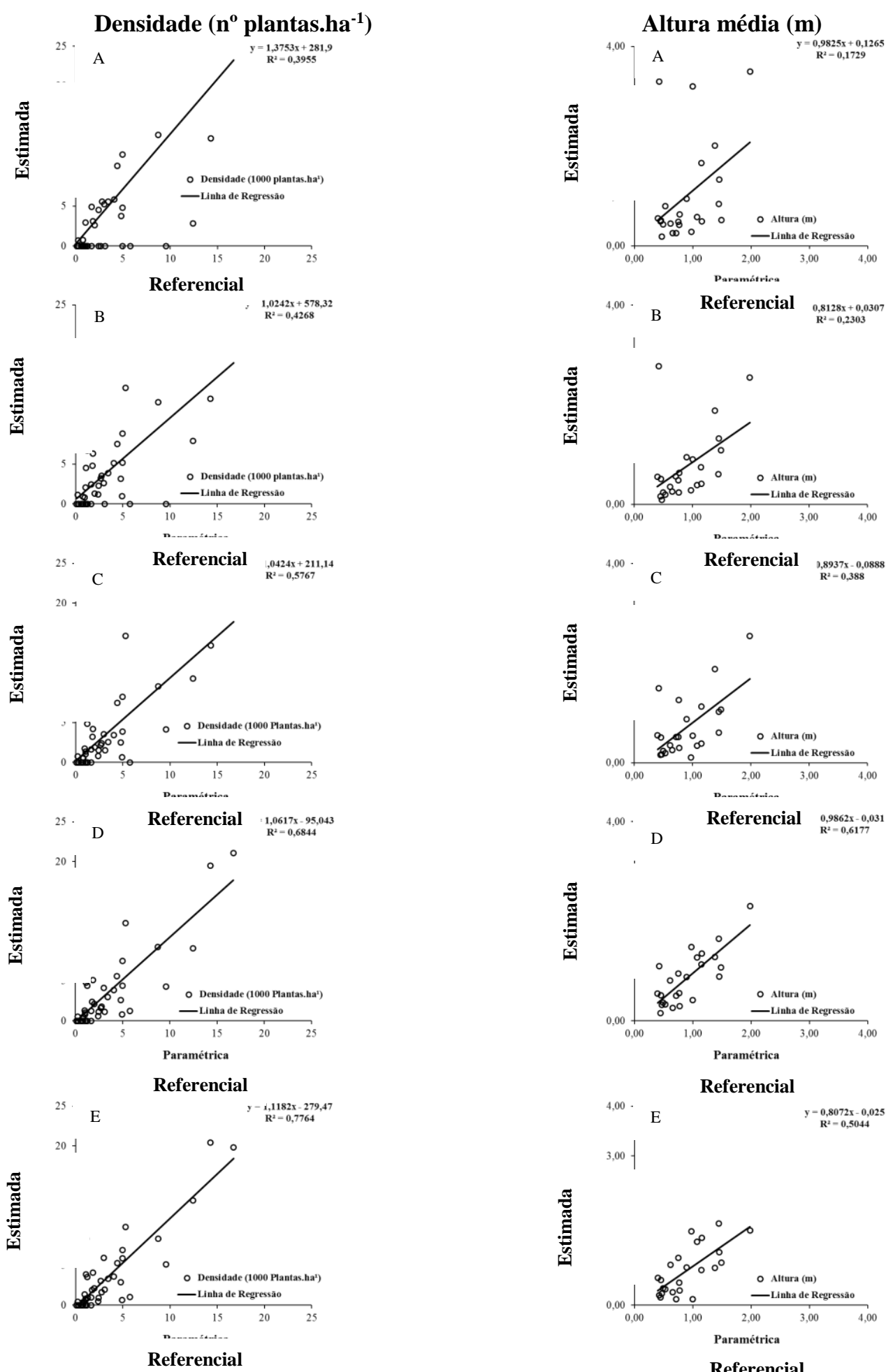

FLORESTA, Curitiba, PR, v. 48, n. 2, p. 265-276, abr/ jun.2108 Araujo. E. J. G et.al. 
Figura 1. Estimativas realizadas nas variações do método de Strand para as variáveis densidade (plantas.ha-1 ${ }^{-1}$ e altura média (m) nos comprimentos de linha de 1 (A), 2 (B), 3 (C), 4 (D) E 5 m (E).

Figure 1. Estimations by using variations of the Strand sampling method for the variables density (plants.ha ${ }^{-1}$ ) and mean height, considering lengths of line of 1 (A), 2 (B), 3 (C), 4 (D), and $5 \mathrm{~m}(\mathrm{E})$.

Tabela 2. Estatísticas de precisão, exatidão e desempenho na estimativa das variáveis número de plantas por hectare e altura média, pelas variações do método de amostragem de Strand.

Table 2. Accuracy statistics and performance of estimation of density and mean height by using variations of the Strand sampling method.

\begin{tabular}{|c|c|c|c|c|c|c|}
\hline \multirow{2}{*}{ Método Strand } & EMA & $\mathbf{W}$ & $\mathbf{R}^{2}$ & $\mathbf{R}$ & $\mathbf{C}$ & \multirow{2}{*}{ Desempenho } \\
\hline & \multicolumn{5}{|c|}{ Densidade (Número de Plantas Por Hectare) } & \\
\hline $\mathrm{L}=1 \mathrm{~m}$ & 3.744 & 0,6558 & 0,3955 & 0,6289 & 0,4124 & Regular \\
\hline $\mathrm{L}=2 \mathrm{~m}$ & 2.542 & 0,7631 & 0,4268 & 0,6533 & 0,4986 & Regular \\
\hline $\mathrm{L}=3 \mathrm{~m}$ & 1.962 & 0,8470 & 0,5767 & 0,7594 & 0,6432 & Bom \\
\hline $\mathrm{L}=4 \mathrm{~m}$ & 1.770 & 0,8948 & 0,6844 & 0,8273 & 0,7403 & Bom \\
\hline $\mathrm{L}=5 \mathrm{~m}$ & 1.515 & 0,9252 & 0,7764 & 0,8811 & 0,8152 & Ótimo \\
\hline \multicolumn{7}{|c|}{ Altura (m) } \\
\hline $\mathrm{L}=1 \mathrm{~m}$ & 0,5822 & 0,5387 & 0,1729 & 0,4158 & 0,2240 & Ruim \\
\hline $\mathrm{L}=2 \mathrm{~m}$ & 0,4439 & 0,6597 & 0,2303 & 0,4798 & 0,3166 & Ruim \\
\hline $\mathrm{L}=3 \mathrm{~m}$ & 0,4219 & 0,7488 & 0,3880 & 0,6229 & 0,4664 & Regular \\
\hline $\mathrm{L}=4 \mathrm{~m}$ & 0,2696 & 0,8700 & 0,6177 & 0,7859 & 0,6837 & Bom \\
\hline $\mathrm{L}=5 \mathrm{~m}$ & 0,3406 & 0,7925 & 0,5044 & 0,7102 & 0,5628 & Regular \\
\hline
\end{tabular}

Em que: EMA é o erro médio absoluto; $W$ é o índice de exatidão de Willmott; $\mathrm{R}^{2}$ é o coeficiente de determinação do ajuste da regressão linear simples para os valores estimados em função dos parâmetros; $R$ é o coeficiente de correlação linear simples; $C$ é o valor da associação entre $W$ e $R$ retratando o desempenho.

As estimativas realizadas pelas variações do método de Prodan para a variável número de plantas por hectare e altura constam na Tabela 3. Na sequência, constam os valores estimados em função dos valores paramétricos do número de plantas por hectare e altura média (Figura 2), bem como as estatísticas de desempenho das variações do método de Prodan (Tabela 4).

Tabela 3. Estatísticas da amostragem realizada pelas variações do método de Prodan para as variáveis número de plantas.ha $\mathrm{h}^{-1}$ e altura média.

Table 3. Statistics for the variations of Prodan sampling method to estimate tree density and mean height.

\begin{tabular}{|c|c|c|c|c|c|c|}
\hline \multirow{2}{*}{ Método Prodan } & Média & Mínimo & Máximo & Desvio & Intervalo & \multirow{2}{*}{ Referencial } \\
\hline & \multicolumn{5}{|c|}{ Densidade (Número de Plantas Por Hectare) } & \\
\hline 1 & $9.178 *$ & 4.163 & 14.828 & 1.418 & $7.760 \leq \overline{\mathrm{R}} \leq 10.595$ & \multirow{4}{*}{5.803} \\
\hline 10 & $13.513^{*}$ & 6.213 & 26.622 & 2.437 & $11.075 \leq \overline{\mathrm{R}} \leq 15.949$ & \\
\hline 20 & $12.200 *$ & 6.404 & 20.931 & 1.989 & $10.211 \leq \overline{\mathrm{R}} \leq 14.189$ & \\
\hline 30 & $11.111 *$ & 5.952 & 18.324 & 1.689 & $9.422 \leq \overline{\mathrm{R}} \leq 12.799$ & \\
\hline \multicolumn{7}{|c|}{ Altura $(\mathrm{m})$} \\
\hline 1 & 0,78 & 0,44 & 1,37 & 0,13 & $0,64 \leq \bar{R} \leq 0,90$ & \multirow{4}{*}{0,82} \\
\hline 10 & 0,85 & 0,42 & 1,49 & 0,13 & $0,72 \leq \bar{R} \leq 0,97$ & \\
\hline 20 & 0,80 & 0,46 & 1,38 & 0,13 & $0,67 \leq \bar{R} \leq 0,92$ & \\
\hline 30 & 0,76 & 0,39 & 1,43 & 0,14 & $0,61 \leq \bar{R} \leq 0,90$ & \\
\hline
\end{tabular}

FLORESTA, Curitiba, PR, v. 48, n. 2, p. 265-276, abr/jun. 2018.

Araujo. E. J. G et.al.

ISSN eletrônico 1982-4688

DOI: $10.5380 /$ rf.v48 i 2.55726 


\section{Densidade ( ${ }^{0}$ plantas.ha-1)}
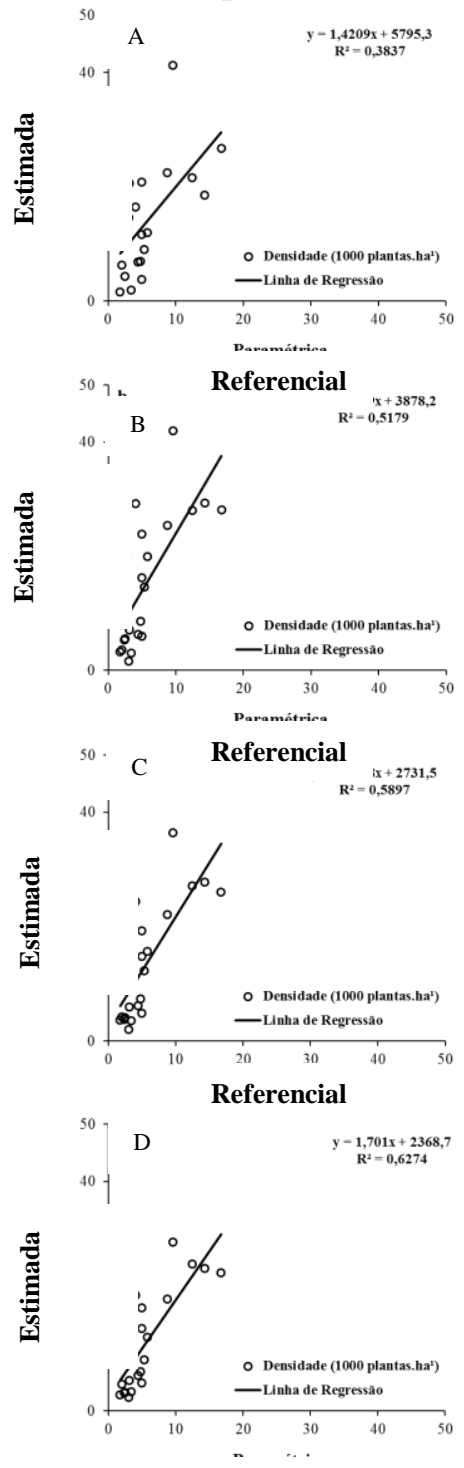
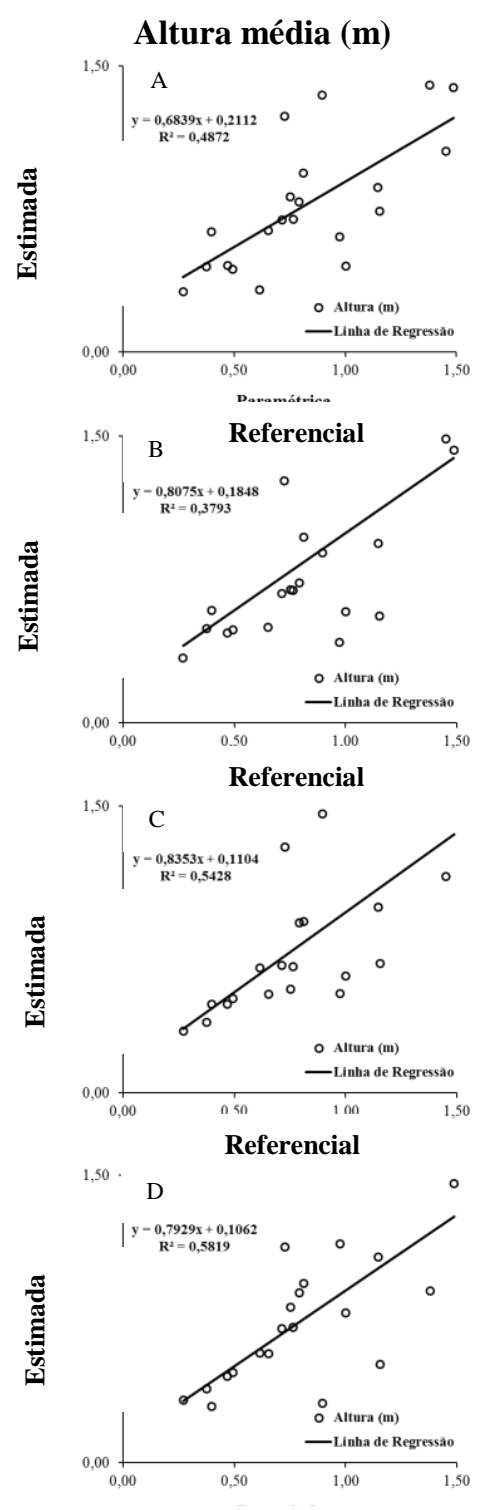

Figura 2. Estimativas Referencial a as variações do método de amostragem de ] Referencial ı variável densidade $\left(\right.$ plantas.ha ${ }^{-1}$ ) e altura média, considerando a $1^{\mathrm{a}}(\mathrm{A}), 10^{\mathrm{a}}(\mathrm{B}), 20^{\mathrm{a}}(\mathrm{C})$, e $30^{\mathrm{a}}(\mathrm{D})$ planta mais próxima do ponto amostral.

Figure 2. Estimations by using variations of the Prodan sampling method for the variables density (plants.ha ${ }^{-1}$ ) and mean height, considering the $1^{\text {st }}(\mathrm{A}), 10^{\text {th }}(\mathrm{B})$, and $30^{\text {th }}(\mathrm{C})$ plant closest to the sampling point.

Tabela 4. Estatísticas de precisão, exatidão e desempenho na estimativa das variáveis número de plantas por hectare e altura média, pelas variações do método de amostragem de Prodan.

Table 4. Accuracy statistics and performance of estimation of density and mean height by using variations of the Prodan sampling method.

\begin{tabular}{|c|c|c|c|c|c|c|}
\hline \multirow{2}{*}{ Método Prodan } & EMA & $\mathbf{W}$ & $\mathbf{R}^{2}$ & $\underline{\mathbf{R}}$ & $\mathbf{C}$ & \multirow{2}{*}{ Desempenho } \\
\hline & \multicolumn{5}{|c|}{ Densidade (Número de Plantas Por Hectare) } & \\
\hline $1^{\mathrm{a}}$ & 8.507 & 0,5001 & 0,3837 & 0,6195 & 0,3098 & Ruim \\
\hline $10^{\mathrm{a}}$ & 9.944 & 0,4724 & 0,5179 & 0,7196 & 0,3400 & Ruim \\
\hline $20^{\mathrm{a}}$ & 8.056 & 0,5487 & 0,5897 & 0,7679 & 0,4214 & Regular \\
\hline
\end{tabular}




\begin{tabular}{ccccccc}
$30^{\mathrm{a}}$ & 6.521 & 0,6214 & 0,6274 & 0,7921 & 0,4922 & Regular \\
\hline $1^{\mathrm{a}}$ & 0,1955 & 0,8308 & 0,4872 & 0,6980 & 0,5799 & Regular \\
$10^{\mathrm{a}}$ & 0,2377 & 0,7778 & 0,3793 & 0,6159 & 0,4790 & Regular \\
$20^{\mathrm{a}}$ & 0,1953 & 0,8542 & 0,5428 & 0,7367 & 0,6293 & Bom \\
$30^{\mathrm{a}}$ & 0,1620 & 0,8672 & 0,5819 & 0,7628 & 0,6615 & Bom \\
\hline
\end{tabular}

Em que: $E M A$ é o erro médio absoluto; $W$ é o índice de exatidão de Willmott; $\mathrm{R}^{2}$ é o coeficiente de determinação do ajuste da regressão linear simples para os valores estimados em função dos paramétricos; $R$ é o coeficiente de correlação linear simples; C é o valor da associação entre $W$ e $R$ retratando o desempenho.

\section{DISCUSSÃO}

As variações do método de Strand, exceto na linha com $1 \mathrm{~m}$ de comprimento, apresentaram estimativas confiáveis para as variáveis densidade (plantas.ha ${ }^{-1}$ ) e altura média (Tabela 1). Como trata-se de avaliação de regeneração natural, a altura das plantas é mensurada com grande precisão. Isso justifica o fato do método de Strand apresentar maiores valores máximos da altura, quando comparado ao método de Prodan.

Com as linhas de 1 e $2 \mathrm{~m}$ de comprimento, os números de plantas por hectare foram superestimados. Linhas de 4 e $5 \mathrm{~m}$ foram as que apresentaram desempenho satisfatório na estimativa dessa variável, propiciando valores muito próximos da média referencial (3.167 plantas.ha ${ }^{-1}$ ). A linha de 5 m estimou 3.262 plantas.ha $^{-1}$, sendo esta a que propiciou a estimativa mais exata. Para a altura das plantas, as linhas de 4 e $5 \mathrm{~m}$ também foram as que propiciaram estimativas exatas. Para linhas de tamanho menor, a média geral da variável tende a ser superestimada.

Percebe-se, com base na Figura 1, que linhas de 1 e $2 \mathrm{~m}$ de comprimento apresentam estimativas muito discrepantes do valor referencial, tornando essas duas condições não exatas, de modo que, em várias UEs, esse tamanho de linha não foi suficiente para amostrar regenerações, o que resultou em estimativas de zero plantas por hectare. $\mathrm{O}$ teste $\mathrm{t}$ para avaliar $\mathrm{H}_{0}: \beta_{0}=0$ foi não significativo ( $\mathrm{p}$-valor $>0,05$ ), indicando que a linha de regressão passa pela origem em todos os comprimentos de linha de Strand. As linhas de 4 e $5 \mathrm{~m}$ apresentaram maior exatidão, com distribuição homogênea dos pontos ao longo da linha de regressão e inclinação próxima de $45^{\circ}$, que é demonstrado pelos valores do coeficiente de inclinação $\left(\beta_{1}\right)$ igual a 1 , conforme o teste t para a significância dos coeficientes (p-valor >0,05 para $\mathrm{H}_{0}: \beta_{1}=1$ ). Além disso, os valores de $\mathrm{R}^{2}$ de 0,68 e 0,77 , respectivamente, retratam precisão na estimativa para esses dois tamanhos de linha.

Para a altura média, as linhas de 1 e $2 \mathrm{~m}$ propiciam subestimativa da altura na maioria dos casos. Contudo, alguns valores com superestimativa conferem à linha de regressão uma inclinação próxima de $45^{\circ}$. A linha de $4 \mathrm{~m}$ propicia os maiores valores de $\mathrm{R}^{2}$, indicando maior precisão, além de apresentar coeficiente de inclinação $\left(\beta_{1}\right)$ mais próximo de 1 . $\mathrm{O}$ teste $\mathrm{t}$ para esse comprimento de linha foi não significativo $(\mathrm{p}$-valor $>0,05)$ para os dois coeficientes, permitindo a aceitação das hipóteses nulas $\left(\mathrm{H}_{0}: \beta_{0}=0\right.$ e $\left.\mathrm{H}_{0}: \beta_{1}=1\right)$.

O desempenho do método de Strand para a variável densidade variou de regular (linhas de 1 e $2 \mathrm{~m}$ ) a ótimo (linha de $5 \mathrm{~m}$ ). Entretanto, já com $3 \mathrm{~m}$ de comprimento da linha de amostragem, obteve-se um desempenho classificado como bom, o mesmo obtido para a linha de $4 \mathrm{~m}$. Para a variável altura, o desempenho foi ruim para linhas de 1 e $2 \mathrm{~m}$ de comprimento, possivelmente por essas propiciarem superestimativas da variável. A linha de $4 \mathrm{~m}$ de comprimento foi a que apresentou o melhor desempenho, com erro médio de estimativa de $0,26 \mathrm{~m}$ (Tabela 2). Destaca-se que a linha de $5 \mathrm{~m}$ de comprimento foi o máximo possível de tamanho de linha a ser avaliado nas condições do presente estudo. Espera-se que, em outras condições, em que seja possível aumentar o tamanho da linha, os resultados provenientes da amostragem sejam mais precisos e exatos.

O método original proposto por Strand (1958) sugere um tamanho de linha equivalente a $5 \pi$, ou seja, 15,7 m (PÉLLICO NETTO; BRENA, 1997). Esses autores ainda destacaram que o método de amostragem em linha reduz o tempo de levantamento em campo e apresenta potencialidade para a avaliação da regeneração natural, pois mantém a mesma base de abordagem para todos os estratos da floresta. No caso da regeneração natural da candeia, o método torna-se viável, pois viabilizou estimativas precisas e exatas com reduzido número de plantas amostradas na linha. Em média, foram amostradas 2 plantas na linha de $1 \mathrm{~m}$ e 5 plantas na linha de $5 \mathrm{~m}$.

No método de Prodan, o valor da média referencial das 21 UE foi 5.803 plantas.ha $^{-1}$. Percebe-se que, em todas as situações, há superestimativa da média referencial, retratando baixa exatidão do método de Prodan para estimar o número de plantas por hectare (Tabela 3). Segundo Kleinn e Vilcko (2006), os métodos que utilizam a k-árvore na estimativa do número de plantas por hectare não são exatos, pois geram erros elevados, principalmente

FLORESTA, Curitiba, PR, v. 48, n. 2, p. 265-276, abr/jun. 2018.

Araujo. E. J. G et.al.

ISSN eletrônico 1982-4688

DOI: $10.5380 /$ rf.v48 i 2.55726 
para menores valores de k, quando o padrão espacial da espécie é agregado. Loetsch et al. (1973) destacaram que os métodos de amostragem que se baseiam nas distâncias entre as plantas propiciam bons resultados somente quando a população apresenta padrão de distribuição espacial uniforme. A explicação é que as distâncias entre plantas são tendenciosas se a população é aleatória ou agregada, pois são poucos os espaços entre as plantas.

Os primeiros trabalhos realizados para avaliar a eficiência desses métodos demonstraram que os resultados não são exatos e bastante tendenciosos quando a população possui padrão espacial de agregação (LOETSCH et al., 1973). Esse resultado foi confirmado pelo teste de Kruskal-Wallis, que retratou diferenças significativas do valor estimado para cada variação do método em relação ao paramétrico ( $\mathrm{p}$-valor $<0,05$ ).

É possível observar que, da primeira para a segunda planta mais próxima do ponto amostral, ocorre um aumento da estimativa de 9.178 para 15.858 plantas.ha $^{-1}$. Um dos fatores que afeta a precisão de uma metodologia de amostragem é o padrão espacial da espécie em questão (SILVA et al., 2008; KLEINN; VILCKO, 2006). Esta pode ser a explicação para os resultados obtidos, pois o padrão agregado da regeneração da candeia (SILVA et al., 2008; ARAÚJO et al., 2016) permite que as primeiras plantas estejam muito próximas do ponto amostral, o que influencia na zona de inclusão da planta, que pode ser definida como a área de abrangência de um indivíduo e é o que define a probabilidade de inclusão desse indivíduo na amostragem.

Como a distância da i-ésima planta ao ponto amostral faz parte do denominador do estimador e apresenta valores de baixa grandeza, ela tende a superestimativa em todos os casos. A partir da quarta planta mais próxima, é nítida a tendência de redução da estimativa (Tabela 3), pois plantas mais distantes do ponto amostral promovem aumento no denominador do estimador e, consequentemente, redução do número de plantas estimado.

É importante considerar que distâncias menores que a distância até a sexta planta são mais sensíveis ao tipo de distribuição espacial da população (LOETSCH et al., 1973). O desempenho da amostragem com k-planta depende consideravelmente do padrão espacial da população. Esse desempenho é muito variável entre populações com padrão agregado, uniforme ou aleatório (KLEINN; VILCKO, 2006).

Para o método de Prodan, Péllico Netto et al. (2012) encontraram resultados parecidos para a variável número de plantas.ha ${ }^{-1}$ da espécie Araucaria angustifolia Bert. O. Ktze, que possui padrão espacial variado. Loetsch et al. (1973) reportaram que é inviável definir um estimador do número de plantas que gere resultados satisfatórios para todos os tipos de padrões espaciais, pois cada padrão apresenta características específicas.

Para a variável altura média das regenerações, a estimativa foi exata em todas as variações do método Prodan (Tabela 3). A altura referencial foi de $0,82 \mathrm{~m}$ e as estimativas médias variaram de $0,71 \mathrm{~m}\left(17^{\mathrm{a}}\right.$ planta) até $0,86 \mathrm{~m}$ ( $26^{\mathrm{a}}$ planta). Em todas as situações, a aplicação do teste de Kruskal-Wallis não foi significante ( $\mathrm{p}$ valor $<0,05$ ), indicando que a média estimada é estatisticamente igual a referencial em todos os casos. Assim, quando o objetivo da amostragem for avaliar a variável altura das regenerações, o método de Prodan pode ser aplicado normalmente e com baixa intensidade amostral, propiciando estimativas confiáveis.

Percebe-se, pela projeção da linha de regressão (Figura 2), que a tendência das variações do método de Prodan é superestimar o número de plantas.ha ${ }^{-1}$, assim como foi verificado nos resultados obtidos por Kleinn e Vilcko (2006). Isso é comprovado pelos elevados valores do coeficiente de inclinação $\left(\beta_{1}\right)$ da reta, que foram próximos a 2 na maioria dos casos, o que implica em uma inclinação consideravelmente superior à desejável $\left(45^{\circ}\right)$, confirmada pela rejeição de $\mathrm{H}_{0}: \beta_{1}=1$ para todas as variações do método de Prodan.

Observando os valores de $\mathrm{R}^{2}$ da linha de regressão, verifica-se que podem ser de até 0,62 para a $30^{\mathrm{a}}$ planta. Entende-se que o método é preciso, ou seja, propicia estimativas que são próximas entre si. Contudo, as estimativas são sempre superiores ao valor referencial, caracterizando a baixa exatidão. Além disso, o erro sistemático, que é retratado pela significância do coeficiente linear ( $\mathrm{p}$-valor $<0,05$ para $\mathrm{H}_{0}: \beta_{0}=0$ ), apresenta-se muito elevado, podendo ser de 5.795 plantas.ha ${ }^{-1}$, quando considera-se a $1^{\mathrm{a}}$ planta mais próxima do ponto amostral.

Para Péllico Netto e Brena (1997), uma das maiores desvantagens do método de Prodan é que seus estimadores podem gerar tendenciosidade quando as plantas apresentam padrão agregado dentro do povoamento, o que corrobora os resultados encontrados neste estudo para a variável número de plantas por hectare.

A linha de regressão para os valores estimados em função dos referenciais apresenta inclinação próxima a $45^{\circ}$ (Figura 2), retratando exatidão na estimativa da altura. Considerando a $20^{\mathrm{a}}$ planta mais próxima do ponto amostral, a estimativa alcança o coeficiente de inclinação $\left(\beta_{1}\right)$ mais próximo de 1 ( $\mathrm{p}$-valor $>0,05$ para $\mathrm{H}_{0}: \beta_{1}=1$ ),

Para a densidade, percebe-se que o desempenho foi classificado como ruim na maioria dos casos e, somente a partir da $20^{a}$ planta mais próxima do ponto amostral, apresentou resultado classificado como regular. Para a variável altura, os resultados foram mais otimistas quanto ao desempenho (Tabela 4).

A partir também da $20^{a}$ planta, o desempenho foi classificado como bom. Os valores dos índices de Willmott (W) e de correlação linear (R) foram de 0,85 e 0,73 , respectivamente. Esses valores sugerem exatidão e 
precisão, respectivamente, quando são amostradas, pelo menos, 20 plantas mais próximas do ponto amostral. Kleinn e Vilcko (2006) reportaram que não é o desempenho da amostragem com k-plantas que será determinante, mas a praticidade de implantação da unidade amostral em condições de campo. Segundo esses autores, o método deve ser testado para obtenção de conclusões definitivas, sendo este tema de pesquisa a ser aprofundado no campo da amostragem em regeneração natural. A intensidade amostral deve ser considerada, pois se for muito alta, mesmo reduzindo a variância, pode elevar os custos e ser inviável.

\section{CONCLUSÕES}

- Dentre as metodologias de amostragem de área variável avaliadas, o método de amostragem de Strand, com linhas de 3 a $5 \mathrm{~m}$ de comprimento, é recomendado para ser aplicado em regeneração natural da espécie Eremanthus erythropappus em áreas submetidas ao sistema de manejo com porta sementes. Tal método gera estimativas precisas e exatas de altura média e número de plantas por hectare.

- No método de Prodan, o padrão espacial agregado da regeneração natural inviabiliza a sua aplicação, pois gera superestimativa do número de plantas por hectare nesses casos. O método somente poderá ser utilizado se o objetivo for amostrar a variável altura média das plantas. Para isso, deve-se considerar, no mínimo, as 20 plantas mais próximas do ponto amostral.

\section{REFERÊNCIAS}

ARAÚJO, E. J. G.; SCOLFORO, J. R. S.; MELLO, J. M.; SÉ, D. C.; OLIVEIRA, A. D.; MORAIS, V. A.; ALTOÉ, T. F.; SCOLFORO, H. F. Sustentabilidade do manejo da candeia nativa (Eremanthus erythropappus (DC.) MacLeish). In: SCOLFORO, J.R.S.; OLIVEIRA, A.D.; DAVIDE, A.C. O manejo sustentável da candeia: o caminhar de uma nova experiência florestal em Minas Gerais. Lavras: Ed. UFLA, 2012, 329 p.

ARAÚJO, E. J. G; PÉlliCO NETTO, S.; MACHADO, S. A.; DALlA CORTE, A. P.; SCOLFORO, J. R. S.; DAVID, H. C.; MORAIS, V. A. Função k de Ripley e índice de Moran na distribuição espacial da regeneração natural da candeia em áreas manejadas. Revista Brasileira Biometria, Lavras, v.34, n.3, p.468-488, 2016.

CARVAlHO, L. G.; OliVEIRA, M. S.; ALVEAS, M. C.; VIANELlO, R. L.; SEDIYAMA, G. C.; NETO, P. C.; DANTAS, A. A. A. Clima. In: SCOLFORO, J. R.; CARVALHO, L. M. T.; OLIVEIRA, A. D. Zoneamento Ecológico-Econômico do Estado de Minas Gerais: Componente geofísico e biótico.Lavras: UFLA, 2008,161 p.

HIRATA, T. Height estimation through Bitterlich's method. Journal of the Physical Society of Japan, Japan, v.37, n.11, p.479-480, 1955.

KERSHAW JR., J. A.; DUCEY, M. J.; BEERS, T. W.; HUSCH, B. Forest Mensuration. Wiley-Blackwell, John Wiley \& Sons, 5th Edition. 2017, 630 p.

KLEINN, C.; VILCKO, F. A new empirical approach for estimation in k-tree sampling. Forest Ecology and Management, v.237, n.1-3, p.522-533, 2006.

LOETSCH, F.; ZOHRER, F.; HALLER, K. E. Forest Inventory. Wien: BLV Verlagsgesellschaft Munchen. 1973, 469 p.

MELO, L. A.; DAVIDE, A. C.; TEIXEIRA, L. A. F. Metodologia para resgate de matrizes e enraizamento de estacas de Eremanthus erythropappus. Cerne, Lavras, v. 18, n. 4, p. 631-638, 2012.

MORI, C. L. S. O.; MORI, F. A.; MENDES, L. M. Caracterização anatômica, química e física da madeira de candeia (Eremanthus erythropappus (DC.) MacLeish). Cerne, Lavras, v. 16, n. 4, p. 451-456, 2010.

OLIVEIRA, G. M. V.; MELLO, J. M.; LIMA, R. R.; SCOLFORO, J. R. S.; OLIVEIRA, A. D. Tamanho e forma de parcelas experimentais para Eremanthus erythropappus. Cerne, Lavras, v. 17, n. 3, p. 327-338, 2011.

PAES, J. B.; FONSÊCA, C. M. B.; LIMA, C. R.; SOUZA. A. D. Eficiência do óleo de candeia na melhoria da resistência da madeira de sumaúma a cupins. Cerne, Lavras, v. 16, n. 2, p. 217-225, 2010.

FLORESTA, Curitiba, PR, v. 48, n. 2, p. 265-276, abr/jun. 2018.

Araujo. E. J. G et.al.

ISSN eletrônico 1982-4688

DOI: $10.5380 /$ rf.v48 i 2.55726 
PÉREZ, J. F. M.; SCOLFORO, J. R. S.; OLIVEIRA, A. D.; MELLO, J. M.; BORGES, L. F. R.; CAMOLESI, J. F. Sistema de manejo para a candeia - Eremanthus erythropappus (DC.) MacLeish - a opção do sistema de corte seletivo. Cerne, Lavras, v. 10, n. 2, p. 257-273, 2004.

PÉLLICO NETTO, S.; BRENA, D. A. Inventário florestal. Curitiba, 1997, 316 p.

PÉlliCO NETTO, S.; ORELlANA, E.; STEPKA, T. F.; LIMA, R.; FIGUEIREDO FILHO, A. Comportamento probabilístico dos raios das sextas árvores no método de Prodan e estimativas dos parâmetros dendrométricos para Araucaria angustifolia (Bertol.) Kuntze nativa. Scientia Forestalis, v.40, n.96, p.517-524, 2012.

PIMENTEL-GOMES, F. Estatística Experimental. Piracicaba: ESALQ. 2009, 451 p.

PRODAN, M. Punktstichprobe fur die forsteinrichtung. Forest. U. Holzwirt, v.23, n.11, p.225-226, 1968.

R DEVELOPMENT CORE TEAM. R: A language and environment for statistical computing. Vienna, Austria: R Foundation for Statistical Computing. Disponível em: <www.R-project.org> Acesso em: 10/11/2011.

SCOLFORO, J. R. S.; OLIVEIRA, A. D.; SILVA, C. P. C.; ANDRADE, I. S. CAMOLESI, J. F; BORGES, L. F. R.; PAVAN, V. M. M. O manejo da candeia nativa. Lavras: Editora UFLA, 2008. 44 p. Boletim Técnico.

SILVA, M. A.; MELLO, J. M.; SCOLFORO, J. R. S.; CZANCK JÚNIOR, L.; ANDRADE, I. S.; OLIVEIRA, A. D. Análise da distribuição espacial da candeia (Eremanthus erythropappus (DC.) MacLeish) sujeita ao sistema de manejo porta-sementes. Cerne, Lavras, v. 14, n. 4, p. 311-316, 2008.

SANTOS, R. C.; MENDES, L. M.; MORI, F. A.; MENDES, R. F. Aproveitamento de resíduos da madeira de candeia (Eremanthus erythropappus) para produção de painéis cimento-madeira. Cerne, Lavras, v. 14, n. 3, p. 241-250, 2008.

SANQUETTA, C. R.; CORTE, A. P. D.; RODRIGUES, A. L. WATZLAWICK, L. F. Inventários Florestais: Planejamento e Execução. 3a . Edição. Curitiba: Autores, 2014. v. 1. 406p.

SCOLFORO, J. R. S.; OLIVEIRA, A. D.; DAVIDE, A. C. O manejo sustentável da candeia: o caminhar de uma nova experiência florestal em Minas Gerais. Lavras, Editora: UFLA. 2012, 329 p.

SILVA, M. A.; MELlO, J. M.; SCOLFORO, J. R. S.; CZANCK JÚNIOR, L.; ANDRADE, I. S.; OLIVEIRA, A.D. Análise da distribuição espacial da candeia (Eremanthus erythropappus (DC.) MacLeish) sujeita ao sistema de manejo porta-sementes. Cerne, Lavras, v.14, n.4, p.311-316, 2008.

STRAND, L. Sampling for volume along a line. Meddelelser Fra Det Norske Skogforsoksvesen, v.51, p.327$331,1958$.

WILLMOTT, C. J. On the validation of models. Physical Geography, v.2, n.2, p.184-194, 1981. 